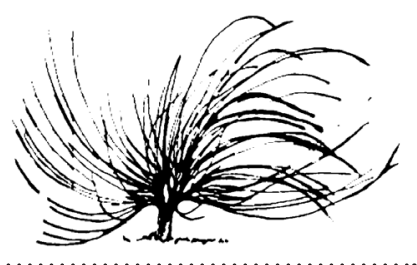

\title{
Liderazgo intersubjetivo: Hacia una emancipación educativa
}

\author{
Libia Mijares Luna ${ }^{1}$ \\ Centro de Investigaciones Culturales y Estudios Lingüísticos \\ San José, Costa Rica \\ mijareslibia@gmail.com
}

\begin{abstract}
Resumen
El liderazgo intersubjetivo promueve una emancipación educativa. El presente ensayo busca posicionarse crítica y reflexivamente en cuanto a la figura del liderazgo en nuestras tareas pedagógicas, para el abordaje de las realidades educativas que enfrentamos como docentes. De tal forma que se fundamenta en la horizontalidad y en la intersubjetividad como aspectos claves para una modificación de las estructuras de poder dominantes que no escapan al currículo educativo, a las escuelas, a las universidades y a cada contexto socio-educativo que se hace eco de la regulación social tradicional. De manera que se promueven algunas herramientas pedagógicas para el abordaje científico de nuestra praxis docente, en el cual la figura de un liderazgo intersubjetivo coadyuva hacia una real participación de los agentes sociales involucrados en el hecho educativo.
\end{abstract}

Palabras clave: Intersubjetividad, liderazgo, currículo educativo, sistematización de experiencias.

\section{(ब) $(\Theta \Theta$}

Recibido: 20 de enero de 2016-Aprobado: 25 de abril de 2016

1 Profesora de español como segunda lengua, Centro de Investigaciones Culturales y Estudios Lingüísticos. San José, Costa Rica. Licenciada en Educación, Universidad Central de Venezuela. Máster en Educación con énfasis en Aprendizaje del Español como Segunda Lengua. Pendiente graduación (mayo 2016) Universidad Nacional. Heredia, Costa Rica. 


\begin{abstract}
Inter-leadership promotes educational emancipation. This essay seeks to position itself critically and reflectively about the figure of leadership in our pedagogical tasks for addressing educational realities. It is based on horizontality and intersubjectivity as key aspects for achieving change in the dominant power structures that do not escape the educational curriculum, schools or universities, and each social context that echoes traditional social regulation. This way, it aims to promote some pedagogical tools to address our educational realities, in which the figure of inter-leadership contributes towards a real participation of the social agents involved in the educational process.
\end{abstract}

Keywords: intersubjectivity, leadership, educational curriculum, systematization of experiences

\title{
Introducción
}

La educación es un proceso que requiere reflexión en el marco de cada contexto particular en el cual se lleve a cabo. A fin de cuentas, como docentes, quienes hacemos vida en este proceso, constituimos entes con formas singulares de percibir la realidad, derivadas de nuestras posturas axiológicas y ontológicas en un nivel consciente o inconsciente.

Cuando se trata de llevar a cabo la tarea docente, surgen las tensiones propias de la dialéctica y la praxis pedagógica; y esto se traduce en un concierto de voluntades, deseos y actos, que son expresiones de una cultura específica de los sujetos socio-históricos que juegan un rol fundamental en el proceso educativo.

Entonces, hallarse en un escenario socio-educativo con múltiples miradas implica también tomar consciencia de que el hecho de la existencia de diversas perspectivas supone también que cada sujeto socio-histórico que forma parte del proceso educativo, constituye un elemento clave que, en definitiva, le aporta un determinado significado al hecho social, histórico, educativo y cultural desarrollado en el contexto profesional docente. 
Con base en esta realidad y necesidad imperativa de reflexión pedagógica sobre nuestras prácticas docentes, el presente ensayo busca reivindicar el concepto de liderazgo académico, para que se asuma este desde una posición de horizontalidad en las relaciones presentes en aquellos escenarios socio-educativos que diariamente protagonizamos. Y significa que cada agente social que forma parte de nuestros particulares ámbitos socio-culturales, socio-históricos y socio-educativos es producto de una historia, de una vida y de una coyuntura singular que, a su vez, es resultado de una realidad multidimensional; por lo que la contribución que hacen los sujetos cuando se involucran en el hecho socio-educativo es determinante para la construcción de la idiosincrasia y concreción de dicho entorno, y ese aporte debe ser valorado.

\section{Liderazgo intersubjetivo}

La búsqueda de reivindicación del concepto de liderazgo académico desde una perspectiva de horizontalidad conlleva a la reflexión sobre nuestras propias construcciones, conocimientos y creencias, lo cual amplía nuestra perspectiva sobre el rol que cumplimos como líderes académicos.

Si nos constituimos agentes sociales que diariamente influenciamos a otras personas, pues esto nos atañe, en el sentido de la necesidad de reflexionar en la forma de percibirnos como seres humanos, llamados a desenvolvernos en el marco de relaciones socio-culturales; sin, por supuesto, dejar de lado que esta realidad expresa la relación inherente que tenemos con nuestros semejantes.

Desde esta perspectiva de horizontalidad, se asume una tarea pedagógica de transformación constante, una en la que cada actor social constituye un elemento clave del proceso educativo. Tal como señala Mencía (2009):

La acción educativa, en este caso la que se lleva a cabo en el aula, es intrínsecamente humana, en la cual no se pueden separar el objeto que es aprehendido por el sujeto cognoscente y en esa interacción dialógica se funden en un mismo propósito el docente y el discente para tratar de ver la realidad desde diferentes perspectivas con la certeza de llegar a un consenso sobre esa misma realidad cuya razón se fundamenta en el respeto mutuo. Esta aspiración no excluye el 
conflicto que podría estar presente en cualquier tipo de relaciones sociales y más aún en la complejidad del aula de clase. (p. 44)

De tal modo que, tomando en cuenta la perspectiva del autor, la búsqueda de reivindicar el proceso educativo (quebrando estructuras tradicionales y positivistas) hacia una real valoración de las características humanas como constructoras de conocimiento implica verse con nuevos ojos, es decir, ampliar nuestras miradas hacia un real reconocimiento del otro u otra y la posibilidad de un encuentro dialógico e indispensable para que se lleve a cabo el aprendizaje. Se recobra, entonces, el sentido de la educación como un proceso eminentemente humano y de objetivos comunes, que no son más que desentrañar la realidad y construir un conocimiento desde diversas posturas y panoramas.

Y sobre la base de este argumento de reconocimiento del otro individuo, ¿qué implicaría la figura de liderazgo? Al respecto, Hernández (2013) menciona que "un líder es aquel capaz de influir sobre los demás, y que por lo tanto ejerce el liderazgo" (p. 88). Pero al mismo tiempo, la autora señala que ningún liderazgo es impositivo y menos coercitivo, porque de ese modo ya perdería su real sentido y significado, dejando de ser liderazgo. Por lo que esa influencia que la autora menciona debe gozar de ciertas características tanto del contexto donde tiene lugar como de aquellas perspectivas axiológicas y ontológicas de sus participantes.

De esa manera, la forma como cada participante asume su rol en el proceso educativo y las consecuencias que esto trae en la praxis pedagógica pasa por contemplar que, en medio de la intersubjetividad, estamos construyendo paulatinamente conocimiento en el marco de una comunidad educativa.

Se aboga por un liderazgo basado en relaciones intersubjetivas que conlleven a una construcción de significados en el marco de la interculturalidad y horizontalidad. Fisher y Strauss (1988, citados por Hernández, 2013, p. 89) sostienen que "los líderes deben comprender a sus seguidores, y para alcanzar esa comprensión necesitan estar suficientemente integrados". De tal forma que se valora el carácter educativo de las relaciones humanas, siempre que estas respondan a las necesidades y a los contextos de los sujetos involucrados.

De esa manera, el presente ensayo rescata la figura de liderazgo en el marco de relaciones intersubjetivas que, en definitiva, son las 
constructoras de conocimiento. $\mathrm{Y}$ en educación, si nos asumimos como pedagogogas o pedagogos críticos, es dentro de nuestros propios escenarios docentes donde podremos realmente reivindicar el liderazgo horizontal y compartido. Un liderazgo que busque respuestas a las problemáticas contextuales que vivimos en nuestras instituciones y que procure una real participación de los sujetos sociales involucrados en la tarea educativa, lo cual generará un conocimiento crítico con miras a la modificación de las estructuras de poder, que les permita a las personas comprometidas ser parte de la toma de decisiones y, en definitiva, contribuir hacia una emancipación educativa que genere una mejora de los procesos pedagógicos que se atienden desde lo interno de cada contexto docente.

Es vital comprender que la tarea de transformación educativa, por un lado, no se puede generar aisladamente; pero, por el otro, tampoco se puede imponer. Estos procesos pedagógicos requieren de una real participación de los sujetos involucrados; pero, ¿bajo qué condiciones podrían lograrse las metas propuestas?, ¿se hablaría de una meta unilateral o de metas compartidas?, ¿cómo gestionar los procesos pedagógicos hacia una real transformación constante de los procesos educativos?

Tal como apunta Hernández (2013), para un liderazgo horizontal se necesita integración. Sin embargo, esto no supone que las personas miembros del proceso deban coincidir en cada aspecto, perspectiva y postura epistemológica, pues de esa manera peligraría la democracia, y estos procesos de pedagogía crítica entrañan procesos de participación democrática.

La lucha docente desde el sector universitario es clave y, al mismo tiempo, constituye un reto pedagógico. Foucault (1984, citado por Mencía, 2009), sostiene que:

La cultura y el poder en el Currículo Nacional están íntimamente relacionados, el cual funciona como un mecanismo de control político del conocimiento, ya que el papel del Estado en la escolarización es emplear la Educación como uno de los mecanismos reguladores. (p. 143)

Como personal pedagogo crítico, estamos llamados a romper estructuras preconcebidas del poder, estructuras que asumen la educación como escolarización y homogeneización colectiva y que, en definitiva, no han reconocido su carácter dialéctico y transformador. 
De tal manera que nuestra tarea como docentes es quebrar dichas estructuras heredadas del positivismo. ¿Seguiremos doblegándonos ante una escolarización que responde a las necesidades socio-económicas y políticas dominantes? O, por el contrario, ¿asumiremos la labor pedagógica en un marco de una intersubjetividad, donde la figura del liderazgo horizontal y gestora de procesos será fundamental para el logro de las metas propuestas en consonancia con una educación humanizada, tendiente a desarrollar el potencial creativo de cada ser humano en el marco de una sociedad democrática?

La labor docente y pedagógica en el ámbito de relaciones intersubjetivas implica que la investigación educativa conlleva una resignificación de las prácticas docentes, entendiendo que la tarea pedagógica es compartida y que cada actor del proceso tiene un conocimiento valioso que aportar. No olvidemos que todo aquello que nos rodea influye de alguna forma en nuestro aprendizaje y, por lo tanto, constituye elemento clave y materia prima de nuestro ser.

En educación, como sujetos críticos es fundamental la objetivación de las propias prácticas docentes. Investigar es una tarea inherente a nuestra praxis, de lo contrario ¿cuál sería el cauce de nuestras inquietudes, críticas y eventuales propuestas pedagógicas? De esa manera se complejiza este aspecto.

Por consiguiente, cuando asumimos el quehacer pedagógico de manera conjunta, estamos reconociendo su carácter complejo, lo que nos conducirá a la elección de metodologías, técnicas e instrumentos investigativos de corte dialéctico que permitirán un abordaje crítico y una objetivación de nuestra tarea educativa, hacia la construcción de un conocimiento realmente endógeno y transformador.

El proceso educativo es de descubrimiento y, en ese sentido, el ser humano es un aprendiente de por vida. Como docentes, somos aprendices permanentes, para quienes la tarea transformadora requerirá de una postura crítica ante la realidad, de reflexión y análisis tanto individual como colectivo. Y es así como la figura de la persona gestora de procesos pasa a ser un elemento dinamizador de la práctica investigativa y de abordaje de la realidad.

En la tarea pedagógica, vista como proceso de análisis y transformación de las realidades educativas, es fundamental la gestión de procesos participativos, horizontales e intersubjetivos. Al respecto Florez (1999), basándose en tesis de epistemologías interaccionistas, menciona 
características inherentes al ser humano, que abarcan aptitudes comunicativas que facilitan un entendimiento lingüístico entre sujetos, así como un mecanismo de coordinación de las acciones desempeñadas. Es decir, el lenguaje (a favor o en contra) como un medio de entendimiento mutuo para negociar definiciones de la situación objeto de estudio.

Asimismo, el autor señala que "es la intersubjetividad la última instancia donde se confronta y se configura el saber humano" (p. 11). A partir de ella, se proponen criterios de verdad o criterios de racionalidad por medio de los cuales los sujetos interactúan en función de los planes de acción:

- $\quad$ Pretensión de verdad: Expresarse comprensiblemente.

- Pretensión de autenticidad: Se habla con la verdad.

- Pretensión de corrección: Se habla según las reglas comunes del lenguaje.

Por lo anterior, se valora aquí el carácter sociocultural del ser humano como una condición clave para el aprendizaje y la construcción de conocimiento. Razón por la cual en educación cada vez se aboga más por la sinergia en nuestras instituciones, reconociendo que solo con la unión podremos enfrentar las propias situaciones derivadas de la praxis docente y así proponer cambios.

De esta forma, tal como lo señala el autor, aunque cada actor social tenga su particular postura paradigmática para el abordaje de la realidad, es ese componente personal e intersubjetivo el que intervendrá en el diálogo por medio de las competencias comunicativas.

A continuación se mencionan algunos procesos inherentes a la intervención pedagógica y cómo a través de un liderazgo intersubjetivo se pueden construir conocimientos realmente emancipadores y transformadores de nuestras prácticas docentes.

\section{Evaluación curricular}

La evaluación educativa debe estar intrínsecamente relacionada con la investigación. Es, pues, la primera, el mecanismo que regula y reorienta nuestra praxis docente. Sin embargo, es esencial reflexionar sobre la postura evaluativa que estamos asumiendo, pues de ello se deriva, si realmente este proceso nos está conduciendo a un conocimiento 
de nuestra propia práctica, hacia a una transformación constante en virtud de la mejora de los procesos educativos que atendemos.

Al respecto, Brovelli (2001) define la evaluación intrínseca del currículo educativo como "evaluar la distancia entre el diseño curricular como norma y lo que realmente ocurre en el proceso de desarrollo curricular y los resultados producidos" (p. 112).

Desde este contexto, los diversos actores que dan vida al proceso educativo son quienes asumen un protagonismo esencial, pues serán quienes tengan a cargo el objetivar su praxis, conociendo profundamente el resultado de sus acciones, gracias a la evaluación.

Este tipo de abordaje curricular debe realizarse en conjunto, donde intervengan todos los actores del proceso educativo en cuestión, de modo que a través del uso de instrumentos pedagógicos se genere un conocimiento científico de la realidad, que le aporte autonomía al personal docente en el desarrollo curricular, ya que dicha comprensión sobre la realidad le brindará información para saber cuándo usar un modelo pedagógico y no otro, y cuándo realizar adecuaciones curriculares de acuerdo con determinado contexto socio-educativo que se atienda.

Pinto (2012) sostiene que, cuando un docente reflexiona sobre su propia praxis educativa, avanza en su profesionalidad y podrá tomar decisiones idóneas en cuanto al currículo que ejecuta; y tal como sostiene Jara (2012) en cuanto a esa reflexión profesional, en ella es posible "encontrar las respuestas o alternativas ante tantas preguntas formuladas" (p. 21).

Es valioso que el personal docente emplee metodologías de investigación de corte dialéctico en el abordaje curricular e implemente instrumentos de índole cualitativa, con el fin de acercarse científicamente a los diversos objetos de estudio (programas, diseños, institución, entre otros) para conocer sus implicaciones pedagógicas, culturales y sociológicas en el marco del desarrollo curricular de cada institución educativa en particular.

Un ejemplo de lo dicho anteriormente lo constituye la evaluación curricular orientada por la crítica artística, la cual contempla que, a través de la aplicación simultánea de diversos instrumentos de evaluación cualitativa, es posible valorar objetos de estudio que forman parte del currículo educativo en el marco de un contexto socio-educativo concreto. 
La evaluación curricular desde una perspectiva cualitativa se puede fundamentar en la triangulación de técnicas e instrumentos de evaluación, entre los que destaca la observación participante.

Es importante acotar que no son más que el personal docente y los sujetos actores del proceso educativo quienes poseen el suficiente conocimiento fáctico de su experiencia, y los que desde una postura protagónica podrán teorizarla, construyendo líneas endógenas de corte propositivo. Además, en la tarea pedagógica es esencial reconocer el saber práctico que poseen los diversos actores del proceso educativo (estudiantes, docentes, personal directivo), el cual es relevante al momento de evaluar un currículo.

Otras técnicas de evaluación, como la revisión documental de programas educativos, pueden ayudar a realizar valoraciones importantes, plasmándolas en diversos instrumentos cualitativos de observación (listas de cotejos, escalas de estimación) de las cuales partir para realizar análisis interpretativos de lo valorado en el currículo institucional.

En el marco de una evaluación curricular, cabe destacar lo propuesto por Ruiz (1999) en cuanto a la evaluación de programas educativos:

1. El programa en sí mismo: el contenido, la calidad técnica, la evaluación, adecuación al contexto.

2. El programa en su desarrollo: relativo a su puesta en marcha.

3. Evaluación final del programa: relativo a la medida y los logros.

Estos tres grandes momentos se contemplan dentro de una evaluación curricular, por el particular hecho de que un programa educativo debe ser evaluado dentro de su marco de acción, produciendo diálogo entre participantes con miras a una mejora de la acción educativa. En ese sentido, al percibir la evaluación curricular como un proceso participativo, la figura de un liderazgo intersubjetivo en el cual la gestión de procesos es clave para una real transformación de las prácticas educativas pasa a ser un recurso imprescindible en la tarea pedagógica.

Al respecto, Mencía (2009) sostiene que la práctica pedagógica contribuye a "construir identidades críticas capaces de iniciar procesos de transformación" (p. 24). Es por ello que se hace vital la promoción de un liderazgo intersubjetivo en nuestras instituciones educativas, para reconstruir el acervo cultural que nos constituye como comunidades 
socio-históricas hacia una real emancipación curricular y posterior mejora de los procesos de enseñanza y aprendizaje que atendemos.

\section{Sistematización de experiencias como fuente de conocimiento}

Desde una postura de abordaje dialéctico de la realidad, hablar de sistematización de experiencias implica hablar de una metodología de trabajo que busca, ante todo, crear instrumentos idóneos y procedimientos de análisis que nos ayuden a conocer de cerca nuestras prácticas docentes y aprender de ellas, generando un conocimiento y transformando los procesos educativos en los cuales nos desempeñamos como agentes sociales.

Dicho conocimiento se produce gracias a métodos de trabajo que contribuyen a captar la complejidad y las dinámicas subyacentes de los procesos de enseñanza y aprendizaje que como docentes atendemos; dinámicas que se originan producto de determinadas prácticas educativas, y que son susceptibles de análisis tanto dialéctico como hermenéutico hacia un real conocimiento y transformación de nuestra praxis educativa.

De tal forma que la sistematización de experiencias debe ser un proceso totalmente consustanciado con las necesidades de nuestras instituciones educativas, y esa necesidad subraya que como agentes sociales es vital adentrarnos en nuestras propias prácticas docentes y así conocer las múltiples voces que le dan vida a la realidad educativa que diariamente protagonizamos.

De esa manera, la sistematización de experiencias se convierte en una herramienta altamente pedagógica, porque del aprendizaje generado a partir de su aplicación se pueden hallar recursos, elementos, factores que permitirán con mayores y sólidos argumentos transformar las prácticas docentes en pro de una mejora de los procesos socio-educativos que desarrollamos diariamente.

Así, la sistematización de experiencias desde un enfoque dialéctico pretende teorizar el proceso de transformación educativa que ocurra; en otras palabras, construir un conocimiento desde las particularidades propias de nuestro contexto educativo; cobra relevancia lo señalado por Jara (2012), en cuanto a que el proceso de construcción de conocimiento posterior a una sistematización de experiencias va a permitir una genuina y singular vinculación entre la teoría y la práctica, ya que el conocimiento se produce de manera endógena a través de 
"aproximaciones teóricas teniendo como punto de partida la sistematización de las prácticas educativas" (p. 41).

Dicha metodología de trabajo logra una singular modalidad de producción de conocimiento, surgida del acercamiento entre sus protagonistas (actores del proceso educativo), quienes aportan una mirada crítica a las experiencias reales en las que participan. De acuerdo con Jara (2012), este procedimiento tiene los siguientes objetivos:

- Favorecer el intercambio de experiencias.

- Tener una mejor comprensión del equipo sobre su trabajo.

- Adquirir conocimientos teóricos a partir de la práctica.

- Mejorar la propia práctica.

Al respecto, el autor mencionado señala que:

La práctica es fuente de conocimientos y aprendizajes, de interpretaciones y de teorías, de confluencias y de conflictos, de aprendizajes y desaprendizajes. No se trata de partir de un marco teórico férreo, elaborado a priori, dentro del cual la práctica tiene que calzar de cualquier manera. (p. 57)

Por consiguiente, para el logro de dichos objetivos, se vuelven sustanciales el trabajo en equipo, la reflexión conjunta, la posición ética profesional en el abordaje de la realidad contextual, y la capacidad de responder pedagógicamente a la naturaleza del aprendizaje humano, el cual siempre se desarrolla en horizontalidad e intersubjetividad. No nos excluye, por supuesto, como personal docente, pues estamos llamados al diálogo colegiado en el abordaje de las diferentes problemáticas curriculares de las instituciones educativas en las cuales nos desempeñamos.

\section{Conclusiones finales}

El liderazgo intersubjetivo constituye un ejercicio poderoso para la transformación educativa, ya que nos conduce a reconocernos como sujetos socio-culturales capaces de enfrentar las propias problemáticas educativas de una forma colegiada; proponiendo iniciativas tendientes a la mejora de nuestras prácticas docentes desde lo interno de nuestras instituciones. 
Cada actor del proceso educativo y agente social en particular posee ese bagaje experiencial que le faculta para indagar en su contexto educativo aprendiendo a leer su propia realidad. Ahora bien, lo sustancial aquí es que esta tarea de abordaje dialéctico de la realidad no es posible realizarla de una forma individual.

Se apela, entonces, a la intersubjetividad entre actores del proceso educativo, quienes, con base en iniciativas cónsonas con las reales demandas de la ciudadanía, pueden llegar a un consenso para un aprendizaje transformador que contribuya a su vez al desarrollo de sujetos críticos, reflexivos y participativos en el marco de una real democracia y de educación popular.

Desde la pedagogía crítica, se asume una intencionalidad política tendiente a una real participación ciudadana en los procesos socio-históricos que se llevan a cabo en cada contexto particular de su realidad pedagógica. Por consiguiente, la emancipación educativa implica una postura crítica y reflexiva ante la realidad, condición coadyuvante para una modificación de las estructuras de poder, gracias a la influencia que como agentes sociales ejercemos sobre nuestros semejantes, en el marco de una democracia participativa que valora a la otra persona, y de un entendimiento intercultural al cual todos los seres humanos estamos llamados.

Por lo tanto, una real participación ciudadana necesita de docentes con compromiso. Más allá de las metodologías y técnicas que podamos usar para abordar nuestros contextos educativos, se hace esencial marcar una sólida postura paradigmática ante la realidad, que nos ayude a aproximarnos pedagógicamente a las diversas situaciones y problemáticas educativas que enfrentamos. Igualmente, no olvidar que, también, la clave para un liderazgo intersubjetivo es atrevernos a participar y reconocer que somos sujetos socio-culturales llamados a aprender con otros en el marco de la democracia.

\section{Referencias bibliográficas}

Bonilla, L. (2014, 26 de junio). Foro "La educación que tenemos y la que queremos". Diarios Últimas Noticias. Recuperado de: http:// www.ultimasnoticias.com.ve/noticias/ciudad/educacion/en-vivo---un-realiza-foro-este-jueves-sobre-calida.aspx

Brovelli, M. (2001). Evaluación curricular. Fundamentos en Humanidades Universidad Nacional de San Luis, 2(4), 101-122. 
Florez, R. (1999). Evaluación pedagógica y cognición. Bogotá, Colombia: Mc Graw Hill.

Hernández, M. L. (2013). Liderazgo académico. Revista de la Educación Superior, 42(3), 105-131.

Jara, O. (2012). La sistematización de experiencias. Práctica y teoría para otros mundos posibles. San José: Centro de Estudios y Publicaciones Alforja.

Mencía, P. (2009). Las relaciones de poder entre docente-alumnos y alumnas en un aula urbana de $5^{\text {to }}$ grado del distrito educativo 0803. Santiago, República Dominicana (Tesis de doctorado). Universidad Estatal a Distancia. Santiago, República Dominicana.

Pinto, R. (2012). Principios filosóficos y epistemológicos del ser docente. $1^{\circ}$ Ed. San José, Costa Rica: Coordinación Educativa y Cultural Centroamericana.

Ruiz, J. (1999). Cómo hacer una evaluación de centros educativos. Madrid: Marcea. 